\title{
LAYERED CODING WITH GOOD ALLOCATION OUTPERFORMS MULTIPLE DESCRIPTION CODING OVER MULTIPLE PATHS
}

\author{
Vu Thanh Nguyen, Ee Chien Chang, and Wei Tsang Ooi \\ Department of Computer Science, \\ National University of Singapore \\ \{nguyenvu, changec, ooiwt\}@comp.nus.edu.sg
}

\begin{abstract}
Packet loss is a serious problem that severely affects the quality of multimedia streaming over error-prone networks. To reduce the variability of packet loss and delay, packets can be transmitted over different network paths (path diversity), after being coded by error-concealment source coding methods like Multiple Description Coding (MDC) or Layered Coding (LC). Researches in this area lead to a common belief that MDC is better than LC when the network conditions (packet loss rate, bandwidth) are grave [1, 2]. However, in this paper, we show that the decision of which packets to send over which paths can greatly affect the performance of LC and MDC, therefore the quality of the streams received. Particularly, using our analytical framework and polynomial algorithms for finding optimal packet allocations, we show that LC outperforms MDC under various critical network conditions.
\end{abstract}

\section{INTRODUCTION}

Packet loss during transmission affects the quality of multimedia streaming over error-prone networks. To handle this problem, we can employ joint source-channel coding techniques, e.g. Multiple Description Coding (MDC) or adding error resilience to Layered Coding (LC), then send packets over multiple paths.

MDC is a form of scalable coding which allows recovery of the first $k$ packets as long as any $k$ packets are received. The more packets received, the better the quality of the stream. Meanwhile, layered or multi-resolution coding (scalable, embedded, progressing coding) like JPEG-2000, MPEG-4 partition the video source data into a base layer and a few enhancement layers with different priorities. The base layer contains the most important video data and decoding only this layer can provide an acceptable perception quality. The enhancement layers deliver complementary information to combine with the base layer for offering higherquality video output. MDC can be obtained by adding redundancies to an existing LC. Therefore intuitively, working directly on LC may be more effective than using MDC.

Traditionally, a single routing path between a sender and a receiver is used for point-to-point, real-time video and audio communication over the Internet. The quality of service of the communication, therefore are subjected to the properties of the path. Events such as bursty loss and occasional congestion, can have negative effects on the quality of the communication. A new model for communication, called packet path diversity has been proposed recently [3]. This model proposes using multiple paths between the sender and the receiver for data transmission. By routing data through multiple disjoint paths, we can achieve an "average" chan- nel for communication with reduced fluctuations in loss rate and delay, as the probability that losses or congestion occur simultaneously in all paths is smaller.

Three major issues have to solve in path diversity implementation: (i) How to select the disjoint paths? (ii) How to enforce the packets to travel through the selected paths? (iii) How to distribute the packets among the paths? The path selection problem have been extensively studied due to its relevance in telephony and wireless network [4]. Specifying the path for the packets to travel can be done either at the application-level using overlay network or at the network-level using IPv6 loose source routing.

Methods for distributing packets have been proposed in the literature, mostly based on multiple descriptive coding (MDC) [3, 5, 6]. In [3], Apostolopoulos proposed sending two independently decodable streams, consisting of even and odd frames respectively, over two different paths. Liang proposed similar system for voice communication [6] by using encoding schemes proposed in [5]. These earlier schemes do not consider network conditions. Liang et. al. later proposed a scheme that chooses the path to send the next packet based on last packet ACK feedback, which was further developed by Chakareski and Girod [7]. However, a reliable back channel with sufficient short round-trip-delay (RTT) is not always available, and hence may not be suitable for real-time communications. Moreover, back channel is also not applicable in broadcasting or multicast video applications.

Several researches on performance comparisons between MDC and LC over multiple paths have been published $[1,2,8]$, and there is a common belief that LC is worse than MDC when the application requires short delay but networks has long RTT or no feedback channel is available. Another conclusion is that MDC is better than LC at higher packet loss rate. These conclusions are drawn based on current LC-packet distribution methods, in which (i) ACK feedback is always required or (ii) protecting base layer will lead to significant delay.

Our work is based on the following observations:

- MDC incurs high bandwidth and CPU overhead, and may not be suitable for all situations, such as streaming to lowpower devices (e.g. PDA, mobile phone). Under the same bandwidth constraint, we can send more LC packets than MDC.

- Distributing packets encoded with MDC over multiple paths is trivial - since all packets are equally important, we can send any packets along any of the chosen path. Meanwhile for LC, the priority difference between layers allows us to choose which and how packets are sent. This suggests that performance improvement can be achieved if better alloca- 
tion algorithm is used. Furthermore, for certain MDC that is obtained by adding redundancies in an existing LC, distributing the MDC packets equally over that paths is just a special case of distributing the LC packets. Thus, for these MDC working directly on the underlying LC could yield higher performance.

- Packet ACK is not a prerequisite in packet distribution. If the sender can cleverly decide in advance how to send packets based on a limited knowledge of the network conditions and does not have to wait for acknowledge from receiver, the problem of delay disadvantage no longer exists. Moreover, if the sender can assign the important level of packets and send them based on their priority without causing any delay or network modification, the problem of posing unpractical solutions is not an issue.

The goal of this paper is to illustrate that, with good allocation algorithm to distribute packets among the paths, $\mathrm{LC}$ can give better performance than MDC. Our claim, which is contrast to the common belief that MDC is better, is supported by experiments on NS2 with audio data coded by well-known MDC and LC methods.

The rest of this paper is organized as follows. In Section 2, we present the model for packet allocation and optimization problem [9], as well as a dependency model for layered media and its allocation algorithm. The result of experimental comparisons between MDC and LC are shown in Section 3. Finally, we conclude in Section 4.

\section{FRAMEWORK AND FORMULATION}

\subsection{Network Setting}

We consider a network model without feedback channels. We have a single receiver, single sender with a set of $m$ disjoint paths $P=$ $\left\{P_{1}, P_{2}, . ., P_{m}\right\}$ between them. Each path $P_{i}$ is associated with a bandwidth capacity, and an packet loss rate, which are denoted as $B_{i}$ and $p_{i}$ respectively. In this paper, the unit of $B_{i}$ is taken to be the number of packets that can be sent over the time period of a chunk. Note that $B_{i}$ is the bandwidth capacity allocated for the data, and could be constrained by a combination of link capacity, effective TCP-friendly bandwidth and tolerable overhead.

For simplicity, the network model can be simplified by considering only two disjoint paths (see Fig. 1), which has been shown to be sufficient for significant improvement in the quality [3]. It can be shown that in the case of two paths, the optimal packet allocation always allocate as many packets as possible onto the more reliable path. Without loss of generality, we assume $p_{1} \leq p_{2}$. Thus, the bandwidth $B_{1}$ will always be exhausted.

A common loss-model for Internet is the extended Gilbert Model, where the network switches between "good" and "bad" states. In a short period of time, we can assume that the network conditions remain constant. Therefore, a single probability value is sufficient to model the loss rate of the links.

\subsection{General model for $\mathrm{LC}$ allocation}

In this section, we describe how we model and compute the allocation for LC. To send a sequences of packets obtained by a MDC over two paths is quite straightforward, because each packet is supposed to be equally important.

We present the generalized mathematical model for maximizing the gain (thus minimizing expected distortion). In this model,

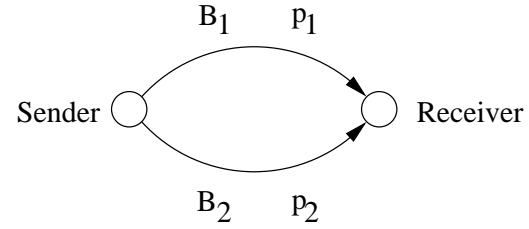

Fig. 1. Network Models

the media data are divided into chunks. A chunk consists of a set of packets, with some interdependencies between them. The interdependencies could be due to layered coding (e.g., between base and enhancement layer) or due to motion estimation (e.g., between I frames and $\mathrm{P}$ frames in MPEG). There are no dependencies among chunks. A chunk is also assumed to be of reasonable length in time (for example, less than acceptable buffering delay). For simplicity, each packet is assumed to be of the same size. Our results can be easily generalized into a model with different packet size.

A chunk is modeled as a graph $G=(V, E)$ where $V$ is the set of packets, and $E$ represents the dependencies between packets there is an edge $(u, v)$ from packet $u$ to packet $v$ if and only if $u$ needs to be received for $v$ to be decoded. We also define $\delta$ to be the tolerable overhead. The total number of packets sent (including FEC packets and duplication packets) must not be greater than $1+$ $\delta$ times the number of packets in $V$.

Define an allocation to be a function $N: V \cup F \times P \rightarrow \mathbb{Z}^{*}$. The number $N(u, i)$ indicates how many times a packet $u$ is sent onto path $P_{i}$. A gain function of a graph $G$ is a function $g_{G}$ : $2^{V} \rightarrow \mathbb{R} . g_{G}(W)$ measures the gain when exactly a subset of packets $W \subseteq V$ are received or recovered by the receiver.

The expected gain of a particular allocation can be calculated as:

$$
E(g)=\sum_{W \subseteq V} g_{G}(W) * \gamma(W, F, N)
$$

where $\gamma(W, F, N)$ is the probability that the receiver receives or recovers exactly the packets in $W$ given a particular allocation $N$ and FEC protection scheme $F$.

The goal now is to maximize $E(g)$ over all possible $N$ and $F$, subjected to the bandwidth constraint, i.e.,

$$
\sum_{u \in V \cup F} N(u, i) \leq B_{i} \text { for all } i
$$

and the overhead constraint, i.e.,

$$
\sum_{i: P_{i} \in P} \sum_{u \in V \cup F} N(u, i) \leq|V|(1+\delta)
$$

Without FEC protection, the probability of successfully receiving a packet $u$ given an allocation $N$ is given by:

$$
\gamma(u, \phi, N)=1-p_{1}^{N(u, 1)} p_{2}^{N(u, 2)}
$$

For brevity, we will use the notation $\gamma_{u}$ to denote $\gamma(u, \phi, N)$ when the context of $N$ is clear. 


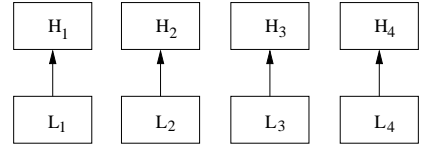

Fig. 2. Packet Pairs Model

\subsection{Layered coding: Pairs model}

Our dependency graph for audio is shown in Fig. 2. In this model, a chunk consists of $K$ pair of packets. Data are divided into two layers. We label the packets in the base layer as $L_{i}$ and packets in enhancement layer as $H_{i}$, where $i=1,2, \ldots K$.

We define the gain function for each pair of packets as follows: If neither packets are received, or only the enhancement packet is received, the gain is 0 . If only the base packet is received, then the gain is $\Delta$. If both the base packet and enhancement packet are received, we let the gain value to be 1 .

The expected gain $E(g)$ at the receiver can be expressed as:

$$
E(g)=\sum_{i=1}^{K}\left(\Delta \gamma_{L_{i}}\left(1-\gamma_{H_{i}}\right)+\gamma_{L_{i}} \gamma_{H_{i}}\right)
$$

We now briefly describe a dynamic programming algorithm for finding optimal allocation $N$ that maximizes $E(g)$ subjected to the bandwidth constraints $B_{1}$ and $B_{2}$. The algorithm works by filling up a 3-dimensional table $A$, where each entry $A_{i}^{m, n}$ stores the optimal expected gain for $i$ pairs of packets given bandwidth constraint $m$ (for $P_{1}$ ) and $n$ (for $P_{2}$ ). Hence the table entry $A_{K}^{B_{1}, B_{2}}$ gives us the maximum expected gain we seek.

We keep another 2-dimensional table $N_{o p t}$ of size $\left(B_{1}+1\right) \times$ $\left(B_{2}+1\right)$, where each entry $N_{o p t}^{m, n}$ keeps the maximum expected gain for a single pair of packets given bandwidth constraint of $m$ (for $P_{1}$ ) and $n$ (for $P_{2}$ ). To initialize each entry in the table, we exhaustively search for all possible allocations. This takes $O(m n)$ time for each entry, giving the total running time for initializing the table $N_{\text {opt }} O\left(B_{1}^{2} B_{2}^{2}\right)$.

Exploiting the recursive nature of Equation 1, the table $A_{i}^{m, n}$ can be filled as follows:

$$
A_{i}^{m, n}= \begin{cases}N_{o p t}^{m, n} & \text { if } i=1 \\ \max _{j, k}\left(A_{i-1}^{m-j, n-k}+N_{o p t}^{j, k}\right) & \text { otherwise }\end{cases}
$$

To find the best allocation for pair $i$, we use dynamic programming technique to trace back all entries for $j$ and $k$. Interested readers are referred to [9].

The recursive step searches through all possible allocations for pair $i$ such that the sum of expected gain for pair $1,2, \ldots i-1$ and pair $i$ is maximum. Therefore, filling in each table entry for $A(i, m, n)$ takes $O(m n)$ time, giving the total running time of this dynamic programming algorithm $O\left(K B_{1}^{2} B_{2}^{2}\right)$. In general, this algorithm is pseudo-polynomial as it depends on the input parameters $B_{1}$ and $B_{2}$. In our case, since $B_{1}+B_{2}$ are bounded by $2 * K(1+\delta)$ and $\delta$ is bounded by a constant in practice, the time complexity of our algorithm is polynomial.

\section{EXPERIMENT}

\subsection{Multiple description and layered coding}

To carry out the experiment, we use a female speech sentence "These days the chicken leg is a rare disk", encoded by PCM at the sampling rate of $8 \mathrm{kHz}$ ( 8 bits/sample) and consisted of 21600 samples ( 2.7 seconds).

To make comparison with the allocation method proposed by Liang in [6], we use the same MDC scheme, which is described in [5]. From each $\mathrm{N}(\mathrm{N}=800)$ original audio samples ( 800 bytes), two MDC packets are constructed. For the first packet, the even original samples are quantized in a finer resolution (PCM, 8 bits per sample) and the difference between adjacent odd and even samples are encoded in coarser resolution (ADPCM, 2 bits per sample). Inversely for the second packet, we encode odd samples in fine resolution and the difference between even and odd samples in coarse resolution. We obtain two MDC stream, each contains 27 packets, each packet has the size of 500 bytes, which means $25 \%$ redundancy excluding packet headers.

As mentioned in Section 2.3, the two LC streams are generated as follow. From each $\mathrm{N}(\mathrm{N}=800)$ original audio samples (800 bytes), the 4 most significant bits (MSB) are extracted and packetized in a base layer packet. The enhancement layer packet consists of 4 least significant bits of $\mathrm{N}$ samples. By this way, each packet has a the size of 400 bytes, but the base packet contributes most important information while the enhancement packet is only useful if its corresponding base packet is received.

For each pair, we compare the quality of encoded packet with original one in terms of Signal-to-Noise ratio (SNR). From that, we find the importance-level ratio between two packets and from the assumption that gain of a pair is 1 , we can calculate the gain of each packet. For MDC, the gain of each packet is around $0.5 \pm$ 0.01 , which is agreeable with the coding nature.

\subsection{Packet allocation schemes over multi paths}

For our experiment, the network model shown in Section 2.1 is used, which is the same model like in $[2,6]$. In these works, Liang and Wang use the same method to send MDC streams, which is simply transmitting one description over one path, and the other description on the other path. Liang assumes both paths having the same bandwidth, therefore for the cases where path's bandwidths are different, we developed an extended version of their scheme. This version, called even allocation scheme, just duplicate packets (from left to right) with the same number of duplication to fulfill the allocated bandwidth on each path.

For LC streams, instead of interleaving the base packets and enhancement packets over two paths as in [2], we employ our proposed scheme in [9], which is briefly described in Section 2.3.

\subsection{Optimal scheme vs. Liang's scheme}

We compare the performance of LC streams sending by our proposed method in [9] with MDC streams sending by Liang's method in [6]. The bandwidth capacity of each path is equal to the 13500 bytes, which is precisely enough for send 27 MDC packets (500 bytes each) on each path. We simulate the transmission using NS2, and for each configuration we conduct the experiment 10 times. The result (average of 10 running times) is show in Fig. 3.

We can easily observe that LC is always better than MDC. It's obvious since LC packet (400 bytes) is smaller than MDC packet (500 bytes), therefore while MDC can send each MDC packet only one times, LC has more room to choose important packets to duplicate. The improvement increases until reaching the peak $(4 \mathrm{~dB})$ when packet loss rate of path 2 is $40 \%$, then decreases. It is because when loss rate is high, the lost of a LC base-layer packet 

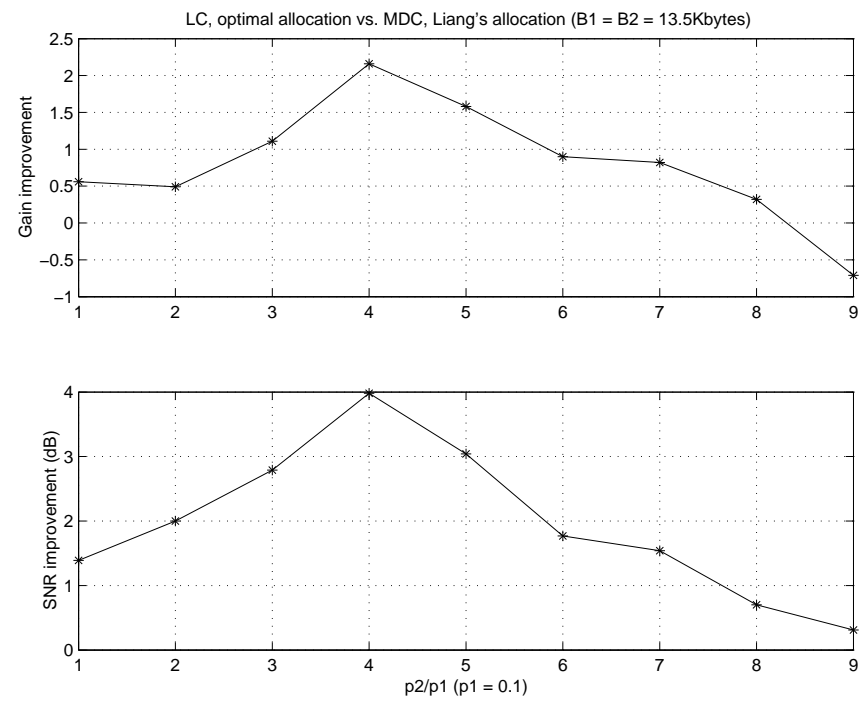

Fig. 3. Improvement of LC with optimal allocation vs. MDC with Liang's allocation. The bandwidth capacity for both paths are the same, B1=B2=13.5 Kbytes (i.e. 5 Kbytes/s if divided to chunk time of 2.7 seconds). The bottom graph shows the SNR of the reconstructed signal as the ratio of the error rate $\mathrm{p} 2 / \mathrm{p} 1$ increases, where $\mathrm{p} 1$ is fixed to be 0.1 . The top graph shows the gain, instead of SNR.

leads to severer quality reduction compared to the lost of a MDC packet.

From the conformation between our gain and SNR measurement showed in Fig. 3, we also see that gain can be directly used to indicate the improvement in signal quality.

\subsection{Optimal scheme vs. Even scheme}

In this part, the performance of LC streams sending by our allocation scheme is compared with MDC streams sending by even distribution scheme. The bandwidths of two paths are varied, as well as their packet loss rates. The result is show in Fig. 4. For all the cases, LC always outperforms MDC, and the improvement decreases as the packet loss rate increases. The quality difference is significant when bandwidths are limited, and lower when more bandwidths are available. That emphasizes the superior of LC to MDC when network conditions are bad.

\section{CONCLUSION}

In this paper, we argue that by using a good algorithm in distributing packets, the quality of received streams can be greatly improved. Moreover, such algorithm does not necessarily require packet ACK feedback, which is a main problem for applying current distribution methods in real time applications. We support our claim by conducting experiment on NS2 tool with audio data coded by well-known MDC and LC methods. The results show that LC can outperform MDC, especially when available bandwidths are scarce.
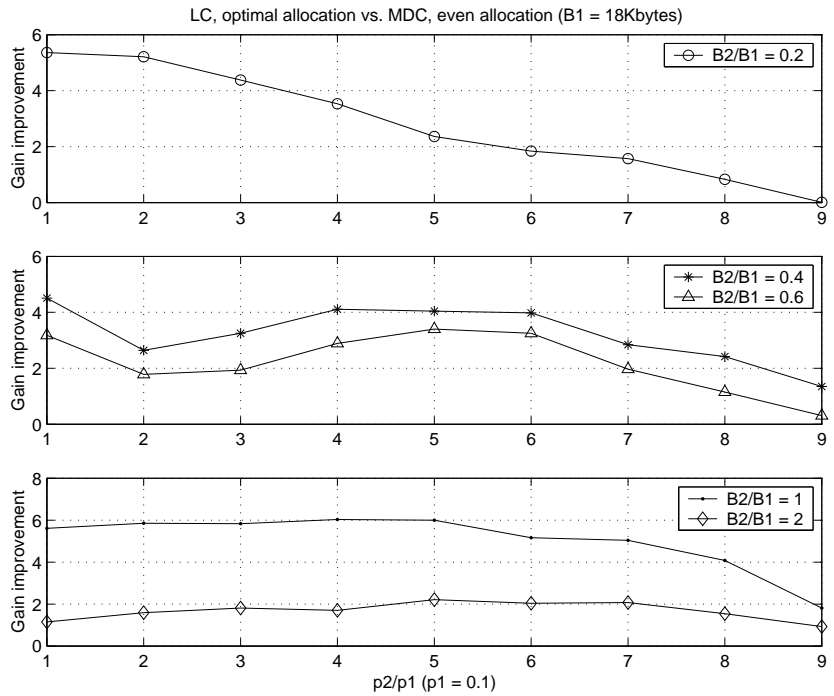

Fig. 4. Improvement of LC with optimal allocation vs. MDC with even allocation.

\section{REFERENCES}

[1] Y. C. Lee, J. Kim, Y. Altunbasak, and R. M. Mersereau, "Performance comparisons of layered and multiple description coded video streaming over error-prone networks," In Proc. IEEE Int. Conf. on Comm., pp. 35-39, 2003.

[2] Y. Wang, S .Panwar, S. Lin, and S. Mao, "Wireless video transport using path diversity: Multiple description vs. layered coding," In ICIP'02, 2002.

[3] John Apostolopoulos, "Reliable video communication over lossy packet networks using multiple state encoding and path diversity," In Proc. of Visual Comm. and Image Processing, VCIP '01, 2001.

[4] D. Sidhu, R. Nair, and S. Abdallah, "Finding disjoint paths in networks," In Proc. of ACM SIGCOMM' 91, 1991.

[5] W. Jiang and A. Ortega, "Multiple description speech coding for robust communication over lossy packet networks," In Proc. of Int. Conf. on Multimedia and Expo, ICME'00, pages 444-447, 2000.

[6] Yi J. Liang, Eckehard G. Steinback, and Bernd Girod, "Realtime voice communication over the internet using packet path diversity," In Proc. of ACM Multimedia 2001, 2001.

[7] Jacob Chakareski and Bernd Girod, "Rate-distortion optimized packet scheduling and routing for media streaming with packet diversity," In Proc. of Data Compression Conf., DCC 2003, 2003.

[8] R. Signh, A. Ortega, L. Perret, and W. Jiang, "Comparison of multiple description coding and layered coding based on network simulations," In Proc. SPIE Conf. on Visual Comm. and Image processing, 2000.

[9] Vu-Thanh Nguyen, Wei-Tsang Ooi, Ee-Chien Chang, "Packet allocation for layer media transmission with path diversity," TR21/03, School of Computing, National University of Singapore, 2003. 